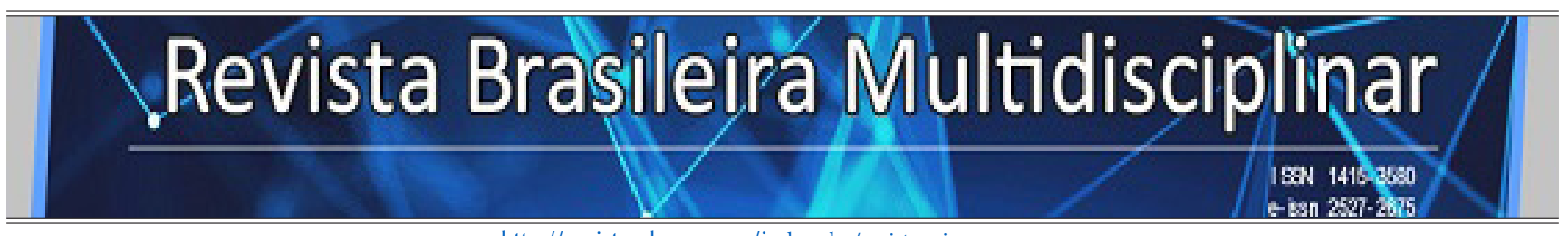

http://revistarebram.com/index.php/revistauniara

\title{
CONHECIMENTO DOS ENFERMEIROS E USO ESCALA DE BRADEN EM UNIDADE DE TERAPIA INTENSIVA: ANÁLISE DA PRODUÇÃo CIENTÍFICA BRASILEIRA
}

\author{
João Victor Batista Cabral*; Lukas Mergulhão de Vasconcelos ${ }^{\star *}$; Martha Maria de Oliveira ${ }^{\star *}$. \\ ${ }^{*}$ Mestre em Ciencias da Saúde..Docente do Centro Universitário da Vitória de Santo Antão - UNIVISA. \\ ${ }^{* *}$ Enfermeiro - Centro Universitário da Vitória de Santo Antão - UNIVISA.
}

*Autor para correspondência e-mail: jvbcabral@gmail.com

\section{Palavras-chave}

Conhecimento

Avaliação

Risco

Unidade de Terapia Intensiva

Lesão por Pressão

\section{KEYWORDS}

Knowledge

Evaluation

Risk

Intensive Care Unit

Pressure Injury

\section{RESUMO}

O objetivo deste estudo foi descrever o conhecimento dos enfermeiros relacionado ao uso da Escala de Braden em Unidade de Terapia Intensiva. Trata-se de uma revisão integrativa descritivo-exploratória, com pesquisa realizada na Biblioteca Virtual em Saúde no período de 2010 a 2017. Os resultados revelaram que a Escala de Braden é um dos instrumentos de avaliação do risco de Lesão por Pressão mais utilizado em Unidade de Terapia Intensiva, sendo objetivo de todos os estudos incluídos na amostra desta pesquisa, porém o conhecimento e uso da escala por enfermeiros ainda é marcado por divergências e heterogeneidade quanto à aplicação e avaliação. Conclui-se que o conhecimento dos enfermeiros sobre a Escala de Braden em Unidade de Terapia Intensiva ainda apresenta fragilidades, sendo considerada sensível e específica na avaliação preditiva, principalmente quando seu escore se aproxima dos valores de maior risco.

\section{NURSING KNOWLEDGE AND BRADEN SCALE USE IN INTENSIVE THERAPY UNIT: AN ANALYSIS} OF BRAZILIAN SCIENTIFIC PRODUCTION

The aim of this study was to describe nurses' knowledge related to the use of the Braden Scale in the Intensive Care Unit. This is an integrative descriptive-exploratory review, with research carried out at the Virtual Health Library from 2010 to 2017. The results revealed that the Braden Scale is one of the most used risk assessment tools for Pressure Injury in the Unit of Intensive Care, being the objective of all the studies included in the sample of this research, however the knowledge and the use of the scale by nurses is still marked by divergences and heterogeneity regarding its application and evaluation. It is concluded that the nurses' knowledge about the Braden Scale in the Intensive Care Unit still has weaknesses, being considered sensitive and specific in the predictive evaluation, especially when its score approaches the highest risk values. 


\section{INTRODUÇÃo}

A Unidade de Terapia Intensiva (UTI) é o ambiente destinado a pacientes em estado crítico que apresentem alterações em um ou mais órgãos vitais, instabilidade hemodinâmica e que necessitam de monitoramento constante e cuidados complexos. Trata-se de uma unidade hospitalar com equipe multiprofissional especializada e que dispõe de tecnologias específicas objetivando dar suporte às funções vitais dos pacientes (DANTAS et al., 2013).

Os pacientes submetidos a cuidados intensivos apresentam, geralmente, alto risco para desenvolver Lesão por Pressão (LPP) devido às limitações ambientais e psicobiológicas, tais como instabilidade hemodinâmica, restrição de movimentos e uso de drogas sedativas e analgésicas, contribuindo para um período prolongado de internação, com comprometimento da percepção sensorial e padrão de mobilidade (GOMES et al., 2011).

Conceitua-se a LPP um dano localizado na pele e/ou tecidos moles subjacentes, geralmente sobre uma proeminência óssea relacionado ao uso de dispositivos de assistência à saúde ou a outros artefatos. A lesão pode se apresentar em pele íntegra ou aberta, ocorrendo como resultado da pressão intensa e/ou prolongada em combinação com o cisalhamento. A tolerância do tecido mole à pressão e ao cisalhamento pode também ser afetada pelo microclima, nutrição, perfusão, comorbidades e condição clínica prévia (NATIONAL PRESSURE ULCER ADVISORY PANEL, 2016).

Estudos em hospitais norte-americanos revelam o aumento do número de internações envolvendo a LPP em aproximadamente 80\% entre 1993 e 2006 (BERLOWITZ, 2016). No Brasil, estima-se que a incidência de LPP nas UTI esteja entre 10,62\% a 62,5\% (ALVES; BORGES; BRITO, 2014). As regiões do corpo mais acometidas por LPP são a sacra, os trocânteres, calcâneos e proeminências ósseas em geral e ocorrem quando a pressão aplicada à pele, por um tempo, é maior que a pressão capilar normal (ROGENSKI; KURCGANT, 2012).

Sobre os fatores de riscos associados à LPP é possível destacar as alterações ou déficits nutricionais, umidade, ventilação mecânica, alterações circulatórias, perfusão tissular alterada, idade, sepse, período prolongado de hospitalização, algumas doenças crônicas como diabetes, nefropatias, hipertensão arterial sistêmica e, principalmente, aumento da exposição à pressão. Observa-se que pessoas com sensibilidade diminuída, imobilidade prolongada e com idade avançada são mais susceptíveis ao desenvolvimento da LPP (SERPA; SANTOS; CAMPONELI; QUEIROZ; 2011).

Em relação ao processo de avaliação e tratamento da LPP, atualmente é possível trabalhar com mais de 40 estilos de ferramentas ou escalas de avaliação do risco, destacando-se como mais utilizadas as escalas de Norton, Warterlow e Braden. É importante ressaltar que a existência de várias escalas está relacionada, sobretudo, às necessidades das distintas áreas clínicas. A Escala de Braden (EB) é a mais empregada mundialmente, sendo recomendada pela Wound Ostomy and Continence Nurses Society e Registered Nurses Association of Ontario/Canadá. Outro fator relevante sobre seu uso é a validação para o português do Brasil, com elevados níveis de sensibilidade e especificidade à avaliação desse risco (COSTA; CALIRI, 2011).

A EB é composta por seis critérios de avaliação: percepção sensorial, grau de umidade, atividade, mobilidade, nutrição, fricção e cisalhamento, com escore definido entre 6 a 23 pontos, podendo variar entre eles, sendo que quanto menor o escore maior será o risco apresentado. Ressalta-se que a estimativa do risco individual deve ser realizada periodicamente, uma vez que o emprego de escalas quantitativas tem a finalidade de manejo preventivo (BAVARESCO; MEDEIROS; LUCENA, 2011).

Frente a essa realidade, a assistência do enfermeiro em UTI tem como objetivo o atendimento ao paciente crítico, compreendendo o diagnóstico de sua condição clínica, intervenções e avaliação dos cuidados específicos de enfermagem, a partir de uma perspectiva humanista voltada para a qualidade de vida, com ênfase no uso do processo de enfermagem como ferramenta de prevenção e promoção à saúde (SILVA; ARAÚJO; OLIVEIRA; FALCÃO, 2010). 


\section{Cabral et al.}

Destaca-se que compete ao enfermeiro a responsabilidade pela gestão do cuidado na tomada de decisões referentes às melhores práticas destinadas à assistência ao paciente (LIMA; CASTILHO, 2015). Pontua-se que o enfermeiro corresponde ao profissional que agrega conhecimentos de medidas preventivas, identificação dos fatores de riscos que contribuem para o desenvolvimento das LPP, e em atuando em conjunto com equipe interdisciplinar desenvolve a prescrição de cuidados para prevenção e tratamento das LPP (SILVA; DICK; MARTINI, 2012).

\section{OвJETIVo}

Assim, considerando a relevância da prevenção de LPP em paciente hospitalizado, este estudo objetivou descrever o conhecimento dos enfermeiros e o uso da Escala de Braden em Unidade de Terapia Intensiva.

\section{Metodologia}

Este estudo fundamentou-se na revisão integrativa descritivo-exploratória, de abordagem qualiquantitativa, realizado através de seis etapas: 1 - Seleção de questão para a revisão; 2 -Seleção dos estudos que compuseram a amostra; 3 - Definição das características primárias que compõem a amostra; 4 - Análise dos achados nos artigos; 5 - Interpretação dos resultados; e, 6 - Relato da revisão, proporcionando avaliação crítica dos achados (MENDES; SILVEIRA; GALVÃO, 2008).

\section{ETAPA 1 - QUestão PARA A ReVisão:}

Como as publicações científicas brasileiras descrevem o conhecimento dos enfermeiros e a utilização da EB no ambiente de UTI?

\section{ETAPA 2 - Seleção dos estudos Que Compuseram A AMOSTRA:}

A coleta de dados para amostra foi realizada a partir de fontes secundárias, por meio de levantamento e análise bibliográfica de publicações realizadas entre os anos de 2010 a 2017, objetivando um recorte atualizado a respeito da temática na Biblioteca Virtual em Saúde (BVS). Os descritores utilizados para pesquisa foram "Conhecimento"; "Avaliação"; "Risco"; "Unidade de Terapia Intensiva" e "Lesão por Pressão", padronizados e presentes nos Descritores em Ciências da Saúde (DeCS) com a utilização do operador booleano and para associação dos descritores.

\section{ETAPA 3 - Definição dAS CARACTERÍsticas PRimárias QUE COMPõe A AMOSTRA:}

Artigos publicados em língua portuguesa, disponíveis on-line de forma gratuita, com texto completo, que atendessem à pergunta norteadora, publicados e indexados na BVS, com os descritores e período selecionado, assim como continham o uso da EB e o conhecimento dos enfermeiros no ambiente de UTI. Os critérios de exclusão foram: trabalhos nomeados como resenhas, noticiários, teses, dissertações, monografias, dossiês, editoriais, capítulos de livros e aqueles que não se enquadrem no recorte temporal estabelecido.

\section{ETAPA 4 - AnÁlise dos achados nos ARTigos:}

Avaliação descritiva após leitura crítica dos resumos de cada artigo e posterior leitura na íntegra, quando coeso com a temática proposta e seus resultados denotassem o conhecimento dos enfermeiros e o uso da EB em UTI no Brasil. Com esses critérios foram levantados 47 artigos, dos quais 6 foram selecionados como amostra.

\section{ETAPA 5 - INTERPRETAÇÃo dOS RESULTADOS:}

Para expressão e interpretação das evidências dos artigos, foi elaborado instrumento (Quadro 1) que 
sintetiza as evidências encontradas com vistas a responder à questão da revisão, com a utilização de pontos descritivos.

\section{ETAPA 6 - RELATO DA REVISÃo:}

Foram categorizadas em tópicos, de acordo com as evidências apresentadas, permitindo construção descritiva respondendo à questão norteadora.

\section{Resultados e discussão}

Entre os seis artigos que compuseram a amostra, no que se refere ao tipo de estudo se verificou que os métodos utilizados foram: descritivo de tipo corte transversal quantitativo (3), exploratório e longitudinal (1), estudo transversal prospectivo (1) e estudo de corte descritivo (1). Sobre a base de dados indexada, quatro estavam no Scientific Electronic Library Online (SciELO) e dois na Base de Dados de Enfermagem (BDENF).

Os artigos foram publicados em periódicos nas regiões Sudeste e Nordeste do Brasil, sendo um nas revistas: Revista da Escola de Enfermagem da Universidade São Paulo (USP), Acta Paulista de Enfermagem, Revista Enfermagem da Universidade Estadual do Rio Janeiro (UERJ), Revista de Enfermagem da Universidade Federal do Piauí (UFPI) e dois na Revista de Enfermagem Universidade Federal de Pernambuco (UFPE).

Em relação ao ano de publicação, foram encontrados dois artigos em 2010, um em 2011, 2014, 2016 e 2017. 


\section{Cabral et al.}

Quadro 1 - Amostra do Estudo - descrição dos estudos de acordo com autor (es), tipo de estudo, base de dados/periódico, ano e evidências.

\begin{tabular}{|c|c|c|c|c|}
\hline Autor (es) & Tipo de Estudo & Periódicos & Ano & Evidências \\
\hline $\begin{array}{l}\text { Valença MP, } \\
\text { Lima PO, } \\
\text { Pereira MM, } \\
\text { Santos RB }\end{array}$ & $\begin{array}{l}\text { Descritivo de } \\
\text { Tipo Corte } \\
\text { Transversal } \\
\text { Quantitativo }\end{array}$ & $\begin{array}{l}\text { BDENF/ Revista de } \\
\text { Enfermagem } \\
\text { UFPE }\end{array}$ & 2010 & $\begin{array}{l}\text { - 73,3\% dos enfermeiros demonstraram não ter } \\
\text { conhecimento acerca das escalas de avaliação de } \\
\text { risco; } \\
\text { - 51,7\% souberam conceituar a LPP; } \\
\text { - 48,3\% não apresentaram conhecimento } \\
\text { satisfatório; } \\
\text { - 93,1\% identificara, as localizações mais comuns à } \\
\text { formação de LPP. }\end{array}$ \\
\hline $\begin{array}{l}\text { Gomes FSL, } \\
\text { Bastos MAR, } \\
\text { Matozinhos } \\
\text { FP, Temponi } \\
\text { HR, Velásquez- } \\
\text { Meléndez G }\end{array}$ & $\begin{array}{l}\text { Descritivo de } \\
\text { Tipo Corte } \\
\text { Transversal } \\
\text { Quantitativo }\end{array}$ & $\begin{array}{r}\text { SciELO/ Revista da Escola } \\
\text { de Enfermagem } \\
\text { da USP }\end{array}$ & 2010 & $\begin{array}{l}\text { - Ocorrência de pelo menos uma LPP por paciente } \\
\text { de 35,2\% (IC 95\%: 27,4-47,7); } \\
\text { - Dos } 50 \text { pacientes portadores de LPP, } 27 \text { (19,0\%) } \\
\text { apresentavam uma única lesão, } 11 \text { pacientes }(7,7 \%) \\
\text { duas lesões, } 12 \text { pacientes }(8,5 \%) \text { três ou mais lesões; } \\
\text { - A pontuação da EB se mostrou fortemente } \\
\text { associada à presença de LPP, sendo mais intensa } \\
\text { nos grupos considerados como risco alto. }\end{array}$ \\
\hline $\begin{array}{l}\text { Araújo TM, } \\
\text { Araújo MFM, } \\
\text { Caetano JA }\end{array}$ & $\begin{array}{l}\text { Exploratório } \\
\text { Longitudinal }\end{array}$ & $\begin{array}{c}\text { SciELO/ Acta Paulista de } \\
\text { Enfermagem }\end{array}$ & 2011 & $\begin{array}{l}\text { - incidência de 59,5\% de LPP; } \\
\text { - As escalas de avaliação de risco de LPP são } \\
\text { ferramentas importantes para o enfermeiro, pois } \\
\text { apontam pontos vulneráveis, reforçam a avaliação } \\
\text { constante e estimulam a prevenção. }\end{array}$ \\
\hline $\begin{array}{l}\text { Barbosa TP, } \\
\text { Beccaria LM, } \\
\text { Poletti NAA }\end{array}$ & $\begin{array}{l}\text { Transversal } \\
\text { Prospectivo }\end{array}$ & $\begin{array}{c}\text { SciELO/ Revista } \\
\text { Enfermagem } \\
\text { UERJ }\end{array}$ & 2014 & $\begin{array}{l}\text { - O uso correto da EB reduz a variação da avaliação } \\
\text { de risco entre enfermeiros, porém é necessário um } \\
\text { treinamento inicial e depois periódico; } \\
\text { - Aponta a necessidade de que os enfermeiros } \\
\text { tenham uma clara compreensão da descrição das } \\
\text { escalas. }\end{array}$ \\
\hline $\begin{array}{c}\text { Braquehais AR; } \\
\text { Dallarosa FS. }\end{array}$ & $\begin{array}{l}\text { Descritivo de } \\
\text { Tipo Corte } \\
\text { Transversal } \\
\text { Quantitativo }\end{array}$ & $\begin{array}{l}\text { SciELO/ Revista de } \\
\text { Enfermagem da } \\
\text { UFPI }\end{array}$ & 2016 & $\begin{array}{l}\text { - 100\% conhecem o termo LPP; } \\
\text { - 100\% conhecem os fatores de risco para LPP; } \\
\text { - Os enfermeiros obtiveram como índice de acertos } \\
\text { global } 72 \% \text {; } \\
\text { - } 10 \% \text { dos enfermeiros referiram participação em } \\
\text { capacitação sobre LPP; } \\
\text { - 85\% dos enfermeiros se sentem preparados para } \\
\text { atuar frente à LPP. }\end{array}$ \\
\hline $\begin{array}{c}\text { Vocci MC, Toso } \\
\text { LAR, Fontes } \\
\text { CMB }\end{array}$ & Corte Descritivo & $\begin{array}{l}\text { BDENF/ Revista de } \\
\text { Enfermagem } \\
\text { UFPE }\end{array}$ & 2017 & $\begin{array}{l}\text { - Com a aplicação da EBQ foi possível identificar o } \\
\text { alto risco de desenvolvimento de LPP }(76,7 \%) ; \\
\text { - Considera-se que ao utilizar um instrumento de } \\
\text { medida, para identificar o risco de desenvolvimento } \\
\text { de LPP em crianças, o enfermeiro contribui para } \\
\text { a construção, o fortalecimento do conhecimento } \\
\text { e do processo de cuidar baseado em evidências } \\
\text { científicas. }\end{array}$ \\
\hline
\end{tabular}

Fonte: Elaboração dos autores, 2019. 


\section{Lesão Por Pressão em Unidade de Terapia Intensiva}

Segundo National Pressure Ulcer Advisory Panel (NPUAP, 2016), LPP é um dano localizado na pele e/ou tecidos moles subjacentes, geralmente sobre uma proeminência óssea ou relacionada ao uso de dispositivo. A LPP é dividida em quatro estágios: (I) pele íntegra com eritema que não embranquece; (II) perda da pele em sua espessura parcial com exposição da derme; (III) perda da pele em sua espessura total e; (IV) perda da pele em sua espessura total e perda tissular. Além disso, outras duas classificações importantes são: LPP não classificável, que é a perda da pele em sua espessura total e perda tissular não visível e LPP tissular profunda, que é a descoloração vermelham escura, marrom ou púrpura, persistente e que não embranquece.

De acordo com pesquisa realizada em Belo Horizonte/MG em UTI foi verificado a ocorrência de pelo menos uma LPP por paciente de 35,2\% (IC 95\%: 27,4-47,7), dos 142 pacientes avaliados 50 eram portadores LPP, $27(19,0 \%)$ apresentavam uma única lesão, 11 pacientes $(7,7 \%)$ duas lesões, 12 pacientes (8,5\%) três ou mais lesões (GOMES et al., 2010).

Dados similares foram encontrados em pesquisa realizada em três UTI em Fortaleza/ CE, dos 42 pacientes avaliados, 25 desenvolveram LPP evidenciando uma incidência de 59,5\%. A detecção das LPP foi identificada nos pacientes entre o $2^{\circ}$ ao $14^{\circ}$ dia de acompanhamento, com tempo médio de surgimento de LPP de 9,6 (DP $\pm 3,3$ ) após internação hospitalar (ARAÚJO; ARAÚJO; CAETANO, 2011).

Tais achados apresentam variações na literatura nacional, demonstrando certa heterogeneidade nos padrões de incidência. Em trabalhos realizados em Porto Alegre/RS, o primeiro, no período de outubro de 2005 a junho de 2006 verificou-se um baixo percentual 1,8\% $(n=27)$ de pacientem com LPP durante o internamento [17]. No outro estudo, as LPP foram evidenciadas em 17 pacientes, perfazendo um total de 22 lesões em diferentes regiões corporais. Verificou-se que 41\% das lesões na região sacral, $32 \%$ nos trocânteres, $18 \%$ no calcâneo, 5\% na escápula e 4\% no maléolo (SILVA; DICK; MARTINI, 2012).

\section{Conhecimento e Uso dA EB POR ENFERmeiros}

A EB é um instrumento que avalia o risco do paciente a desenvolver a LPP, apesar de ser um mecanismo que auxilia à prevenção, muitos enfermeiros não têm conhecimento suficiente sobre a escala. Em pesquisa realizada em um hospital escola do Recife/PE foi evidenciado um percentual significativo $(73,3 \%)$ de enfermeiros que demonstraram não ter conhecimento sobre as escalas de avaliação de risco, sendo identificado que somente $51,7 \%$ dos enfermeiros souberam conceituar a lesão por pressão e $93,1 \%$ citaram as localizações mais comuns à formação da lesão, sugerindo que apesar dos enfermeiros entenderem que os pacientes de risco devem ser avaliados, os mesmos não têm conhecimento de como fazê-lo (VALENÇA; LIMA; PEREIRA; SANTOS, 2010).

No estudo de Braquehais, Dallarosa (2016), foi evidenciado que 100\% dos enfermeiros conhecem o termo LPP e os fatores de risco para o seu desenvolvimento, todavia quando avaliados quanto ao conhecimento acerca da prevenção de LPP em UTI, obtiveram como índice de acertos total $72 \%$. Tal fato pode ser resultante de baixa formação/capacitação, uma vez que apenas $10 \%$ dos enfermeiros referiram participação em capacitação sobre LPP. Porém, mesmo frente a essa realidade os enfermeiros referem se sentir preparados para atuar em situações de pacientes com LPP.

Em pesquisa realizada no Centro de Terapia Intensiva de um hospital do interior de São Paulo, foi observado maior eficácia na avaliação do risco da LPP entre os escores preditivos 14,13 e 12 (COSTA; CALIRI, 2011). No estudo Araújo, Araújo, Caetano (2011), a EB apresentou pontuação média de 11,6 a 12,5, sendo 01 paciente com risco mínimo, 34 com risco moderado e 07 com risco alto. Em outro estudo realizado em quatro UTI de um hospital geral privado da cidade de São Paulo os pacientes avaliados obtiveram a seguinte classificação: baixo risco (30,5\%), risco moderado (40,3\%) e alto risco $(29,2 \%)$.

Vocci, Toso, Fontes (2017) identificaram que com a aplicação da EB adaptada à pediatria foi possível 
identificar o alto risco de desenvolvimento de LPP (76,7\%), nos pacientes da UTI Pediátrica, sugerindo que sua utilização neste ambiente, com as adequadas adaptações a torna um bom instrumento de predição de risco. Resultados semelhantes foram encontrados no estudo de Bevaresco, Medeiros e Lucena (2011), onde o escore médio para o risco de LPP para a primeira avaliação por meio da EB foi de 11,35, sendo o maior escore de 20 e o menor de 07.

As escalas de avaliação de risco de LPP são ferramentas importantes para o enfermeiro, pois apontam pontos vulneráveis, reforçam necessidade de avaliação constante e estimula à prevenção (MENEGON et al., 2012). O conhecimento dos instrumentos de avaliação em qualquer profissão é fundamental para o bom cuidado do paciente. Na enfermagem, especificamente, existem diversas formas de prevenção de riscos, destacando o uso adequado da EB.

Os cuidados de enfermagem abrangem intervenções relacionadas ao acompanhamento integral do paciente em risco de adquirir lesão, por meio das escalas de predição de risco (GOMES et al., 2010). A LPP é um tipo de lesão que pode ser evitada, pois existem medidas preventivas. Entre esses procedimentos, o primeiro é a avaliação de risco, pois permite que os enfermeiros identifiquem os pacientes com maior vulnerabilidade. De acordo com Morita, Poveda, Santos, Marcelino (2012), para que esta avaliação possa ser fidedigna, o uso de um instrumento medidor de risco é essencial, assim como o seu correto e oportuno uso.

Além da avaliação de risco a associação com outras medidas são fundamentais. Ressalta-se, que o uso prévio de uma escala validada e amplamente utilizada fortalece o processo de enfermagem permitindo que a prescrição de cuidados de enfermagem realizada pelo enfermeiro seja voltada de forma integral ao cuidado diário com a pele (hidratação e higienização), mudança de decúbito, mobilização do paciente, com vistas a evitar o posicionamento prolongado do paciente sobre as preeminências ósseas, uso de colchão pneumático, coxins, protetores cutâneos e placa de hidrocoloide.

\section{Possibilidades e Desafios Para o Uso da EB}

Considera-se que ao utilizar um instrumento de medida para identificar o risco de desenvolvimento de LPP o enfermeiro contribui para a construção, o fortalecimento do conhecimento e do processo de cuidar baseado em evidências científicas (VOCCI; TOSO; FONTES, 2017). A EB é muito versátil podendo ser empregada em vários setores no ambiente hospitalar, assim como fora do mesmo, tais como pacientes acamados em domicílio que necessitam de avaliação de risco de LPP constantemente. O uso correto da EB reduz a variação da avaliação de risco entre enfermeiros, porém é necessário um treinamento inicial e depois periódico, uma vez que se verifica a necessidade de que os enfermeiros tenham uma clara compreensão da descrição da escala com a finalidade de melhor execução, reduzindo a-probabilidade de erros na classificação do paciente crítico (BARBOSA; BECCARIA; POLETTI, 2014).

Um dos pontos preocupantes em relação ao uso da Escala de Braden é a falta de concordância dos enfermeiros sobre a classificação de risco da LPP como mostra o estudo realizado no Hospital de Base de São Jose do Rio Preto/SP, que foi realizado em quatro UTI sendo observado diferença na classificação dos pacientes em níveis de risco (SIMÃO; CALIRI; SANTOS, 2013). A falta de concordância pode comprometer o planejamento efetivo do plano de cuidados, pois ele deve estar de acordo com o nível de risco do paciente.

\section{Conclusão}

A incidência de LPP em UTI é variável, entretanto com valores que se destacam na atualidade. Trata-se de um evento evitável, sendo um dos focos da aliança mundial de segurança do paciente e sua prevenção constitui-se como uma das metas do atendimento efetivo e seguro.

O conhecimento dos enfermeiros sobre a EB em UTI pode ser considerado intermediário, todavia 
ressalta-se que pela limitação amostral, tal inferência carece de maior investigação.

Nos estudos levantados foi possível perceber que os enfermeiros demonstraram ter um bom nível de clareza quanto a definição conceitual da LPP e seus fatores de risco, todavia não é suficiente somente o entendimento sobre a lesão e seus fatores genéticos, é necessário ter clara compreensão da descrição das escalas preditivas e assim efetuar seu uso como ferramentas de atendimento ao paciente crítico, pois apontam pontos vulneráveis, reforçam a avaliação constante e estimulam a prevenção.

O uso da EB nos ambientes de UTI no Brasil foi amplamente demonstrado, sendo esta escala considerada sensível e específica na avaliação preditiva, principalmente quando seu escore se aproxima dos valores de alto risco. Sua adaptação à UTI pediátrica também se mostrou viável, com bons índices de identificação do risco.

Sugere-se com esta revisão, que tal temática seja foco de mais estudos, uma vez que a interface entre o conhecimento do enfermeiro e uso da EB em UTI é essencial para o fortalecimento da enfermagem enquanto ciência do cuidado baseada em evidências.

\section{REFERÊNCIAS}

ALVES, A.G.P.; BORGES, J.W.P.; BRITO, M.A. Avaliação do risco para úlcera por pressão em unidades de terapia intensiva: uma revisão integrativa. Jornal de Pesquisa Fundamental Care On line, v.6, n.2., p.793-804, 2014.

ARAÚJO, T.M.; ARAÚJO, M.F.M.; CAETANO, A.J. Comparação de escalas de avaliação de risco para úlcera por pressão em pacientes em estado crítico. Acta Paulista de Enfermagem, v.24, n.5, p.695-700, 2011.

BARBOSA, P.T.; BECCARIA, M.L.; POLETTI, A.A.N. Avaliação do risco de úlcera por pressão em UTI e assistência preventiva de enfermagem. Revista de Enfermagem da UERJ, v.22, n.3, p.353-8, 2014.

BAVARESCO, T.; MEDEIROS, H.R.; LUCENA, F.A. Implantação da Escala de Braden em uma unidade de terapia intensiva de um hospital universitário. Revista Gaúcha de Enfermagem, v.32, n.4, p. 703-10, 2011.

BRAQUEHAIS, A.R.; DALLAROSA, F.S. Conhecimento dos Enfermeiros Acerca da Prevenção de Lesões por Pressão em Unidade de Terapia Intensiva. Revista de Enfermagem da UFPI, v.5, n.4, p. 13-8, 2016.

BERLOWITZ, D. Epidemiology, pathogenesis and risk of pressure ulcers. Up To Date; 2016. Disponível em: http://www.uptodate.com/contents/epidemiology-pathogenesis-and-risk-assessment-of-pressure-ulcers. Acesso em: 02, Dez, 2017.

COSTA, G.I.; CALIRI, L.H.M. Validade preditiva da escala de Braden para pacientes de terapia intensiva. Acta Paulista de Enfermagem, v. 24, n.6, p. 772-7, 2011.

DANTAS, A.L.M.; ARAÚJO, J.D.B.; FERREIRA, P.C.; VALENÇA, C.N.; DINIZ, K.D.; LIRA, A.L.B.C. Prevenção de úlcera por pressão segundo a perspectiva do enfermeiro intensivista. Revista de Enfermagem UFPE on line ,v. 7, n1, p. 706-12, 2013.

GOMES, L.S.F.; BASTOS, R.A.M.; MATOZINHOS, P.F.; TEMPONI, R.H.; MELÉNDEZ, V.G. Avaliação de risco para úlcera por pressão em pacientes críticos. Revista Escola Enfermagem da USP, v. 45, n.2, p. 13-18, 2011. 


\section{Cabral et al.}

GOMES, L.S.F.; BASTOS, R.A.M.; MATOZINHOS, P.F.; TEMPONI, R.H.; MELENDEZ, V.G. Fatores associados à úlcera por pressão em pacientes internados nos Centros de Terapia Intensiva de Adultos. Revista Escola de Enfermagem da USP,v. 44, n.4, p. 1070-6, 2010.

LIMA, A.F.C.; CASTILHO, V. Body mobilization for prevention of pressure ulcers: direct labor costs. Revista Brasileira Enfermagem, v. 68, n. 5, p. 647-52, 2015.

MENDES, S.D.K.; SILVEIRA, C.C.R.P.; GALVÃO, M.C. Revisão integrativa: método de pesquisa para a incorporação de evidências na saúde e na enfermagem. Texto \& Contexto Enfermagem, v.17, n.4, p. 758-64, 2008.

MENEGON, B.D.; BERCINI, R.R.; SANTOS, T.C.; LUCENA, F.A.; PEREIRA, S.G.A.; SCAIN, F.S. Análises das subescalas de Braden como indicativos de risco para úlcera por pressão. Texto \& Contexto Enfermagem, v. 21, n. 4, p.854-61, 2012.

MORITA, S.P.B.A.; POVEDA, B.V.; SANTOS, J.M.; MARCELINO, L.A. Conhecimento dos enfermeiros acerca dos instrumentos de avaliação de risco para úlcera por pressão. Revista REENVAP, v. 02, p. 09$23,2012$.

NATIONAL PRESSURE ULCER ADVISORY. Painel. Disponível em: http:www.npuap.org. Acesso em: 10 mai, 2017.

ROGENSKI, B.M.N.; KURCGANT, P. Incidência de úlceras por pressão após a implementação de um protocolo de prevenção. Revista Latino-Americana de Enfermagem, v.20, n.2., [07telas], 2012.

SERPA, F.L.; SANTOS, G.C.L.V.; CAMPANILI, F.G.C.T.; QUEIROZ, M. Validade preditiva da escala de Braden para o risco de desenvolvimento de úlcera por pressão em pacientes críticos. Revista Latino-Americana de Enfermagem, v.19, n.1., [08 telas], 2011.

SILVA, L.N.W.E.; ARAÚJO, A.R.; OLIVEIRA, C.E.; FALCÃO, L.F.T.V. Aplicabilidade do protocolo de prevenção de úlcera de pressão em unidade de terapia intensiva. Revista Brasileira de Terapia Intensiva, v.22, n.2, p. 175-185, 2010.

SILVA, V.R.M.; DICK, M.R.N.; MARTINI, C.A. Incidência de úlcera por pressão como indicador de qualidade na assistência de enfermagem. Revista de Enfermagem da UFSM, v.2,n.2, p.339-346, 2012.

SIMÃO, F.M.C.; CALIRI, L.H.M.; SANTOS, B.C. Concordância entre enfermeiros quanto ao risco dos pacientes para úlcera por pressão. Acta Paulista de Enfermagem, v. 26, n.1., p.30-5, 2013.

VALENÇA, P.M.; LIMA, O.P.; PEREIRA, M.M.; SANTOS, B.R. Percepção dos enfermeiros sobre a prevenção das úlceras por pressão em um hospital escola da cidade de Recife. Revista de Enfermagem da UFPE on line, v. 4., n. 2,p. 673-82, 2010.

VOCCI, C.M.; TOSO, R.M.L.; FONTES, B.M.C. Aplicação da Escala de Braden em unidade de terapia intensiva pediátrica. Revista de enfermagem da UFPE on line, v.11., n.1., 165-72, 2017.

ZAMBONATO, B.P.; ASSIS, M.C.S.; BEGHETTO, M.G. Associação das sub-escalas de Braden com o risco do desenvolvimento de úlcera por pressão. Revista Gaúcha de Enfermagem, v.34,n. 1., 21-28, 2013. 